\title{
Robust Formation Coordination of Robot Swarms with Nonlinear Dynamics and Unknown Disturbances: Design and Experiments
}

DOI:

10.1109/TCSII.2021.3074705

\section{Document Version}

Accepted author manuscript

Link to publication record in Manchester Research Explorer

Citation for published version (APA):

Hu, J., Turgut, A. E., Lennox, B., \& Arvin, F. (2021). Robust Formation Coordination of Robot Swarms with Nonlinear Dynamics and Unknown Disturbances: Design and Experiments. IEEE Transactions on Circuits and Systems II: Express Briefs, 69(1), 114-118. https://doi.org/10.1109/TCSIl.2021.3074705

\section{Published in:}

IEEE Transactions on Circuits and Systems II: Express Briefs

\section{Citing this paper}

Please note that where the full-text provided on Manchester Research Explorer is the Author Accepted Manuscript or Proof version this may differ from the final Published version. If citing, it is advised that you check and use the publisher's definitive version.

\section{General rights}

Copyright and moral rights for the publications made accessible in the Research Explorer are retained by the authors and/or other copyright owners and it is a condition of accessing publications that users recognise and abide by the legal requirements associated with these rights.

\section{Takedown policy}

If you believe that this document breaches copyright please refer to the University of Manchester's Takedown Procedures [http://man.ac.uk/04Y6Bo] or contact uml.scholarlycommunications@manchester.ac.uk providing relevant details, so we can investigate your claim.

\section{OPEN ACCESS}




\title{
Robust Formation Coordination of Robot Swarms with Nonlinear Dynamics and Unknown Disturbances: Design and Experiments
}

\author{
Junyan Hu, Member, IEEE, Ali Emre Turgut, Barry Lennox, Senior Member, IEEE, \\ and Farshad Arvin, Senior Member, IEEE
}

\begin{abstract}
Coordination of robot swarms has received significant research interest over the last decade due to its wide real-world applications including precision agriculture, target surveillance, planetary exploration, etc. Many of these practical activities can be formulated as a formation tracking problem. This brief aims to design a robust control strategy for networked robot swarms subjected to nonlinear dynamics and unknown disturbances. Firstly, a robust adaptive formation coordination protocol is proposed for robot swarms, which utilizes only local information for tracking a dynamic target with uncertain maneuvers. A rigorous theoretical proof utilizing the Lyapunov stability approach is then provided to guarantee the control performance. Towards the end, real-time hardware experiments with wheeled mobile robots are conducted to validate the robustness and feasibility of the proposed formation coordination approach.
\end{abstract}

Index Terms-Collective behavior, networked systems, formation coordination, robust control, mobile robots, swarm robotics.

\section{INTRODUCTION}

Swarm robotics is the domain by which a large number of mostly homogeneous robots are coordinated to collaboratively complete a desired common task [1]. Swarm robotic systems are usually distinguished by the following features: i) communication of each robot is limited in the sense that only neighboring agents communicate among themselves, ii) all robots in a swarm follow the same set of rules and work in unison to achieve a common goal, and iii) stability of a swarm system will not be affected significantly if some of the agents leave the network [2]. Swarm robotics is being applied in a variety of real-world problems, for instance, autonomous shepherding [3], dynamic mapping [4], cooperative planetary exploration [5], etc.

Control strategies in swarm robotics are primarily inspired from the behavior of natural swarms, for example, swarms of ants, bees, birds and fish (see Fig. 1). As one of the most effective methods to coordinate large-scale robot swarms, distributed formation control becomes a significant research direction in swarm robotics. Based on this technique, some

This work was supported by the Engineering and Physical Sciences Research Council (EPSRC) [grant numbers EP/R026084/1 and EP/P01366X/1] and EU H2020-FET Robocoenosis [grant number 899520].

(Corresponding author: Farshad Arvin)

J. Hu, B. Lennox and F. Arvin are with the Department of Electrical and Electronic Engineering, The University of Manchester, M13 9PL, Manchester, UK. (e-mail: \{junyan.hu, barry.lennox, farshad.arvin\}@manchester.ac.uk)

A.E. Turgut is with the Department of Mechanical Engineering, Middle East Technical University, 06800 Ankara, Turkey
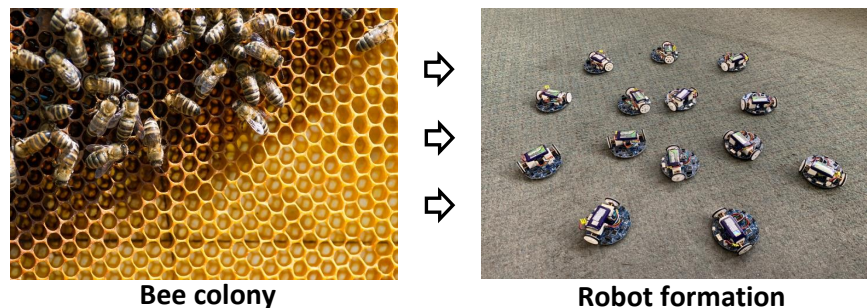

Fig. 1. The idea of robot formation is inspired from the collective behavior of natural swarms, e.g., bee colony.

secondary control objectives can be assigned to cooperative robots, which include distributed source seeking [6], plume front monitoring [7], search and rescue [8], and mobile manipulator motion tracking [9], [10]. The basic form of the formation tracking control is the 'leader-following' case in which the followers attain the desired formation around the leader (or the target) and keep tracking the target. Pioneering research on distributed formation control of swarm of unmanned aerial vehicles (UAVs) was proposed in [11], but only second-order systems were considered and global information of the communication topology (i.e., the Laplacian matrix of the graph) was used in the control protocol design. Hence, the swarm system was not fully distributed. In order to deal with this issue, adaptive formation algorithms for swarm systems were developed, such as [12]. However, the robustness of the swarm system in the presence of unknown disturbances and model uncertainties were not addressed. Besides, the target's uncertain maneuvers was also not considered. Variety of advanced formation control approaches of UAVs and mobile robots have been proposed from a theoretical perspective [13]-[15], but verifying the theory through real-world robotic systems still remains as a challenge.

Motivated by the above rapid progress and challenges in designing control strategies for robot swarms, in this brief, we proposed a novel collective formation coordination method, which is robust to unknown disturbances and uncertain maneuvers of the target. The contributions in this study can be summarized as follows:

- A novel collective formation coordination strategy is developed for networked robot swarms subjected to unknown disturbances with a dynamic target to track.

- Only neighboring robots need to communicate to each other, which significantly reduces the bandwidth require- 
ments.

- The proposed method has been verified via real-time experiments involving a swarm of affordable autonomous mobile robots equipped with low-cost sensors.

\section{PRELIMINARIES AND PROBLEM FORMULATION}

\section{A. Basic concepts on graph theory}

Consider a network graph $\mathcal{G}=(\mathcal{V}, \mathcal{E}, \mathcal{A})$ with a set of nodes and associated edges. An edge starting at the $i^{\text {th }}$ node and ending at the $j^{\text {th }}$ node is represented by $(i, j)$, which means information can flow from node $i$ to node $j$ and thus node $j$ is a neighbor of node $i . a_{i j}$ is the weight of the edge $(j, i)$ and $a_{i j}=1$ if $(j, i) \in \mathcal{E}$. Let $D=\operatorname{diag}\left\{d_{i}\right\}$ with $d_{i}=\sum_{j=1}^{N} a_{i j}$ and the Laplacian matrix $L$ of $\mathcal{G}$ is then calculated by $L=$ $D-\mathcal{A}$. If node $i$ can receive information directly from the root (labelled as 'node 0'), then this node is called a 'pinned' node and an edge $(0, i)$ is generated between them with the weight $g_{i}=1$. The pinning matrix is defined as $G=\operatorname{diag}\left(g_{i}\right)$.

\section{B. Problem description}

Consider a swarm robotic system containing $N$ robots and there is a static or moving target for all the robots to track. For $i \in\{1,2, \ldots, N\}$, the dynamics of the target and robots are described by

$$
\left\{\begin{array}{l}
\dot{x}_{0}(t)=A x_{0}(t)+q\left(x_{0}\right)+B u_{0}(t), \\
\dot{x}_{i}(t)=A x_{i}(t)+q\left(x_{i}\right)+B\left(u_{i}(t)+d_{i}(t)\right),
\end{array}\right.
$$

where $x_{0} \in \mathbb{R}^{n}$ and $x_{i} \in \mathbb{R}^{n}$ are the states of the target and robot $i$ respectively. $u_{0} \in \mathbb{R}^{m}$ denotes the unknown input to the target reflecting its uncertain maneuvers and $u_{i} \in \mathbb{R}^{m}$ is the control input of the robot $i$ to be designed. $d_{i} \in \mathbb{R}^{m}$ is the unknown disturbance signal. $A \in \mathbb{R}^{n \times n}$ and $B \in \mathbb{R}^{n \times m}$ are constant matrices with $\operatorname{rank}(B)=m$ and $(A, B)$ is stabilizable. The nonlinear function $q($.$) is assumed to satisfy$ the following Lipschitz condition:

$$
\|q(x)-q(y)\| \leq \eta\|x-y\| \quad \forall x, y \in \mathbb{R}^{n},
$$

where $\eta$ is the Lipschitz constant.

In the dynamical target tracking system, the unknown input $u_{0}$ and external disturbances $d_{i}$ are parameterized with a set of base functions as

$$
u_{0}(t)=\kappa_{0} \theta_{0}(t), \quad d_{i}=\kappa_{i} \theta_{i}(t),
$$

where $\theta_{0}(t), \theta_{i}(t)$ are the base function vectors and $\kappa_{0}, \kappa_{i}$ are unknown constant matrices to be updated autonomously.

The target collective formation is described by the vector $f(t)=\left[f_{1}^{T}(t), f_{2}^{T}(t), \ldots, f_{N}^{T}(t)\right]^{T}$ with $f_{i} \in \mathbb{R}^{n}$ being a designed guidance signal obtained by the corresponding $i^{\text {th }}$ robot.

This brief mainly solves the following three problems for swarm robotic systems: (i) under what conditions the collective formation coordination can be accomplished subjected to unknown disturbances; (ii) how to construct the coordination protocol to form the target static/dynamic formation; (iii) how to implement the proposed method in real-time hardware experiments.

\section{ROBUST FORMATION CONTROL STRATEGY DESIGN}

In this section, a collective formation coordination strategy is proposed for networked robot swarms.

Since the unknown matrix $\kappa_{0}$ is not available to the robots, the $i^{t h}$ robot estimates the unknown matrices $\kappa_{0}, \kappa_{i}$ by $\hat{\kappa}_{i 0}$, $\hat{\kappa}_{i}$. Motivated by [16], [17], a robust adaptive control protocol with dynamic coupling weights is proposed as follows:

$$
\left\{\begin{aligned}
u_{i} & =c_{i} K \xi_{i}+\gamma_{i}+\hat{\kappa}_{0} \theta_{0}-\hat{\kappa}_{i} \theta_{i} \\
\dot{c}_{i} & =\rho_{i} \xi_{i}^{T} \Gamma \xi_{i} \\
\dot{\hat{\kappa}}_{i 0} & =-\tau_{i} \Xi \xi_{i} \theta_{0}^{T} \\
\dot{\hat{\kappa}}_{i} & =-\sigma_{i} \Xi \xi_{i} \theta_{i}^{T}
\end{aligned}\right.
$$

where $\xi_{i}=\sum_{j=1}^{N} a_{i j}\left(\left(x_{i}-f_{i}\right)-\left(x_{j}-f_{j}\right)\right)+g_{i}\left(\left(x_{i}-f_{i}\right)-\right.$ $\left.x_{k}\right)$ is the formation tracking error, $\rho_{i}, \tau_{i}$ and $\sigma_{i}$ are positive constants. $K, \Gamma$ and $\Xi$ are all constant gains to be chosen properly. $\gamma_{i}$ is a continuously differentiable function to be determined later.

Since matrix $B$ given in (1) is of full rank, we can find a nonsingular matrix $\left[\tilde{B}^{T}, \bar{B}^{T}\right]^{T}$ with $\tilde{B} \in \mathbb{R}^{m \times n}$ and $\bar{B} \in$ $\mathbb{R}^{(n-m) \times n}$ such that $\tilde{B} B=I_{m}$ and $\bar{B} B=0$.

The following Theorem 1 is proposed to construct the coordination protocol and satisfy the aforementioned objectives. All the robots in the swarm are coordinated to maintain a robust configuration around the target based on relative state information.

Theorem 1: Assume that all the robots are connected by a communication network for information exchange and at least one of the robots can detect the states of the target. If the coordination feasibility condition

$$
\bar{B} A f_{i}-\bar{B} \dot{f}_{i}=0, \forall i \in\{1, \ldots, N\}
$$

is satisfied. The formation coordination task can be achieved by the robot swarm on applying the robust coordination protocol provided in (4) with $K=-R^{-1} B^{T} P, \Gamma=P B R^{-1} B^{T} P$, $\Xi=(P B)^{T}$ and $\gamma_{i}=\tilde{B} \dot{f}_{i}-\tilde{B} A f_{i}$, where $P>0$ is the solution of the Linear Matrix Inequality (LMI)

$$
A^{T} P+P A-P B R^{-1} B^{T} P+\mu P P+\frac{\eta^{2}}{\mu}<0
$$

for given $R>0$ and $\mu>0$.

Proof: Let the local coordination error of each follower be defined as $\delta_{i}=x_{i}-f_{i}-x_{0}$. We can get

$$
\dot{\delta}_{i}=A \delta_{i}+B\left(u_{i}+d_{i}-u_{0}\right)+A f_{i}-\dot{f}_{i}+q\left(x_{i}\right)-q\left(x_{0}\right)
$$

Let $\delta=\left[\delta_{1}^{T}, \delta_{2}^{T}, \ldots, \delta_{N}^{T}\right]^{T}$. Then $\xi=\left[\xi_{1}^{T}, \ldots, \xi_{N}^{T}\right]^{T}$ can be expressed in the Kronecker product form as

$$
\xi=\left((L+G) \otimes I_{n}\right) \delta .
$$

Let $\tilde{\kappa}_{j 0}=\hat{\kappa}_{j 0}-\kappa_{0}$ and $\tilde{\kappa}_{j}=\hat{\kappa}_{j}-\kappa_{j}$ for all $j \in$ $\{1, \ldots, N\}$. Define $Q(x)=\left[q\left(x_{1}\right)^{T}, \ldots, q\left(x_{N}\right)^{T}\right]^{T}$ and $Q\left(x_{0}\right)=\left[q\left(x_{0}\right)^{T}, \ldots, q\left(x_{0}\right)^{T}\right]^{T}$. The closed-loop error dy- 
namics $\delta$ embedded with the adaptive coordination protocol (4) can be represented in the compact form given by

$$
\begin{aligned}
\dot{\delta}= & \left(I_{N} \otimes A+\hat{C}(L+G) \otimes B K\right) \delta \\
& +((L+G) \otimes A) f+\left(I_{N} \otimes B\right)\left(\tilde{\kappa}_{0} \Theta_{0}-\tilde{\kappa} \Theta\right) \\
& -\left((L+G) \otimes I_{n}\right) \dot{f}+((L+G) \otimes B) \gamma \\
& +Q(x)-Q\left(x_{0}\right),
\end{aligned}
$$

where $\hat{C}=\operatorname{diag}\left(c_{1}, \ldots, c_{N}\right), \tilde{\kappa}_{0}=\operatorname{diag}\left(\tilde{\kappa}_{10}, \ldots, \tilde{\kappa}_{N 0}\right)$, $\tilde{\kappa}=\operatorname{diag}\left(\tilde{\kappa}_{1}, \ldots, \tilde{\kappa}_{N}\right), \Theta_{0}=\operatorname{col}\left(\theta_{0}, \ldots, \theta_{0}\right), \Theta=$ $\operatorname{col}\left(\theta_{1}, \ldots, \theta_{N}\right)$ and $\gamma=\left[\gamma_{1}^{T}, \gamma_{2}^{T}, \ldots, \gamma_{N}^{T}\right]^{T}$.

Consider the following Lyapunov function candidate:

$$
\begin{aligned}
V= & \delta^{T}((L+G) \otimes P) \delta+\sum_{i=1}^{N} \frac{1}{\rho_{i}}\left(c_{i}-\alpha\right)^{2} \\
& +\sum_{i=1}^{N} \operatorname{tr}\left(\frac{1}{\tau_{i}} \tilde{\kappa}_{i 0}^{T} \tilde{\kappa}_{i 0}\right)+\sum_{i=1}^{N} \operatorname{tr}\left(\frac{1}{\sigma_{i}} \tilde{\kappa}_{i}^{T} \tilde{\kappa}_{i}\right),
\end{aligned}
$$

where $\alpha$ is a positive scalar to be decided later.

Then, the time derivative of $V$ along the trajectory of (9) is given by

$$
\begin{aligned}
\dot{V}= & 2 \delta^{T}((L+G) \otimes P A) \delta+\sum_{i=1}^{N} 2\left(c_{i}-\alpha\right) \xi_{i}^{T} \Gamma \xi_{i} \\
& +2 \delta^{T}((L+G) \otimes P B)\left(\tilde{\kappa}_{0} \Theta_{0}-\tilde{\kappa} \Theta\right) \\
& +2 \delta^{T}((L+G) \hat{C}(L+G) \otimes P B K) \delta \\
& +2 \delta^{T}\left((L+G)^{2} \otimes P A\right) f \\
& -2 \delta^{T}\left((L+G)^{2} \otimes I_{n}\right) \dot{f} \\
& +2 \sum_{i=1}^{N} \operatorname{tr}\left(\frac{1}{\tau_{i}} \tilde{\kappa}_{i 0}^{T} \dot{\tilde{\kappa}}_{i 0}\right)+2 \sum_{i=1}^{N} \operatorname{tr}\left(\frac{1}{\sigma_{i}} \tilde{\kappa}_{i}^{T} \dot{\tilde{\kappa}}_{i}\right) \\
& +2 \delta^{T}\left((L+G)^{2} \otimes P B\right) \gamma \\
& +2 \delta^{T}((L+G) \otimes P)\left(Q(x)-Q\left(x_{0}\right)\right)
\end{aligned}
$$

Note that

$$
2 \delta^{T}((L+G) \hat{C}(L+G) \otimes P B K) \delta=-2 \sum_{i=1}^{N} c_{i} \xi_{i}^{T} \Gamma \xi_{i}
$$

and

$$
\sum_{i=1}^{N} 2 c_{i} \xi_{i}^{T} P B R^{-1} B^{T} P \xi_{i}=2 \sum_{i=1}^{N} c_{i} \xi_{i}^{T} P B R^{-1} B^{T} P \xi_{i} .
$$

Since $\left[\tilde{B}^{T}, \bar{B}^{T}\right]^{T}$ is non-singular, from (5), it can be readily shown that

$$
A f_{i}-\dot{f}_{i}+B \gamma_{i}=0
$$

which can be represented in a compact form

$$
\left(I_{N} \otimes A\right) f-\left(I_{N} \otimes I_{n}\right) \dot{f}+\left(I_{N} \otimes B\right) \gamma=0 .
$$

Pre-multiplying both sides of (15) by $(L+G)^{2} \otimes P$, we have

$$
\begin{array}{r}
\left((L+G)^{2} \otimes P A\right) f-\left((L+G)^{2} \otimes P\right) \dot{f} \\
+\left((L+G)^{2} \otimes P B\right) \gamma=0 .
\end{array}
$$

Substituting (12), (13) and (16) into (11), we get

$$
\begin{aligned}
\dot{V}= & \delta^{T}\left((L+G) \otimes\left(P A+A^{T} P\right)\right. \\
& \left.-2 \alpha(L+G)^{2} \otimes P B R^{-1} B^{T} P\right) \delta \\
& +2 \delta^{T}((L+G) \otimes P B)\left(\tilde{\kappa}_{0} \Theta_{0}-\tilde{\kappa} \Theta\right) \\
& +2 \sum_{i=1}^{N} \operatorname{tr}\left(\frac{1}{\tau_{i}} \tilde{\kappa}_{i 0}^{T} \dot{\tilde{\kappa}}_{i 0}\right)+2 \sum_{i=1}^{N} \operatorname{tr}\left(\frac{1}{\sigma_{i}} \tilde{\kappa}_{i}^{T} \dot{\tilde{\kappa}}_{i}\right) \\
& +2 \delta^{T}((L+G) \otimes P)\left(Q(x)-Q\left(x_{0}\right)\right) .
\end{aligned}
$$

According to the properties $\operatorname{tr}\left(A^{T}\right)=\operatorname{tr}(A)$ and $\operatorname{tr}(A B)=$ $\operatorname{tr}(B A)$, we have

$$
\begin{aligned}
& \delta^{T}((L+G) \otimes P B)\left(\tilde{\kappa}_{0} \Theta_{0}-\tilde{\kappa} \Theta\right) \\
= & \sum_{i=1}^{N}\left(\sum_{j=1}^{N} \hat{L}_{i j} \delta_{j}^{T}\right)(P B) \tilde{\kappa}_{i 0} \theta_{0}-\sum_{i=1}^{N}\left(\sum_{j=1}^{N} \hat{L}_{i j} \delta_{j}^{T}\right)(P B) \tilde{\kappa}_{i} \theta_{i} \\
= & \sum_{i=1}^{N} \operatorname{tr}\left(\tilde{\kappa}_{i 0}(P B)^{T} \xi_{i} \theta_{0}^{T}\right)-\sum_{i=1}^{N} \operatorname{tr}\left(\tilde{\kappa}_{i}(P B)^{T} \xi_{i} \theta_{i}^{T}\right)
\end{aligned}
$$

where $\hat{L}_{i j}$ is the element on the $i^{\text {th }}$ row and $j^{\text {th }}$ column of the matrix $(L+G)$.

The selections of the adaptive terms can be given by

$$
\begin{gathered}
\dot{\tilde{\kappa}}_{i 0}=-\tau_{i}(P B)^{T} \xi_{i} \theta_{0}^{T}, \\
\dot{\tilde{\kappa}}_{i}=-\sigma_{i}(P B)^{T} \xi_{i} \theta_{i}^{T} .
\end{gathered}
$$

Substituting (19) and (20) into (17) gives

$$
\begin{aligned}
\dot{V}= & \delta^{T}\left((L+G) \otimes\left(P A+A^{T} P\right)-2 \alpha(L+G)^{2} \otimes \Gamma\right) \delta \\
& +2 \delta^{T}((L+G) \otimes P)\left(Q(x)-Q\left(x_{0}\right)\right) .
\end{aligned}
$$

Since all the eigenvalues of the symmetric matrix $(L+G)$ have positive real parts, there exists a nonsingular $U$ such that $U^{T}(L+G) U$ is in the diagonal form $J=\operatorname{diag}\left(\lambda_{1}, \ldots, \lambda_{N}\right)$, where $0<\lambda_{1} \leq \lambda_{2} \leq \cdots \leq \lambda_{N}$ are the eigenvalues of $(L+G)$. Let $\Phi=\left[\Phi_{1}^{T}, \ldots, \Phi_{N}^{T}\right]^{T}=\left(U^{T} \otimes I_{n}\right) \delta$, then it follows from (21) that

$$
\begin{aligned}
\dot{V}= & \Phi^{T}\left(J \otimes\left(P A+A^{T} P\right)-2 \alpha J^{2} \otimes \Gamma\right) \Phi \\
& +2 \Phi^{T}\left(J U^{T} \otimes P\right)\left(Q(x)-Q\left(x_{0}\right)\right)
\end{aligned}
$$

According to the Lipschitz condition (2), we have

$$
\begin{aligned}
& 2 \Phi^{T}\left(J U^{T} \otimes P\right)\left(Q(x)-Q\left(x_{0}\right)\right) \\
= & 2 \Phi^{T}(\sqrt{\mu} J \sqrt{\Theta} \otimes P)\left(\frac{1}{\sqrt{\mu}} \sqrt{\Theta}^{-1} U^{T} \otimes I_{n}\right)\left(Q(x)-Q\left(x_{0}\right)\right) \\
\leq & \mu \Phi^{T}(J \Theta J \otimes P P) \Phi+\frac{1}{\mu}\left(\left(U \otimes I_{n}\right)\left(Q(x)-Q\left(x_{0}\right)\right)\right)^{T} \times \\
& \left(\Theta^{-1} \otimes I_{n}\right)\left(\left(U \otimes I_{n}\right)\left(Q(x)-Q\left(x_{0}\right)\right)\right) \\
\leq & \mu \Phi^{T}(J \Theta J \otimes P P) \Phi+\frac{\eta^{2}}{\mu} \Phi^{T}\left(\Theta^{-1} \otimes I_{n}\right) \Phi,
\end{aligned}
$$

where $\Theta>0$ is a diagonal matrix. By selecting $\Theta=J^{-1}$, we can obtain

$$
\begin{aligned}
\dot{V} & \leq \Phi^{T}\left(J \otimes\left(P A+A^{T} P+\mu P P+\frac{\eta^{2}}{\mu}\right)-2 \alpha J^{2} \otimes \Gamma\right) \Phi \\
& \left.\leq \sum_{i=1}^{N} \lambda_{i} \Phi_{i}^{T}\left(P A+A^{T} P+\mu P P+\frac{\eta^{2}}{\mu}\right)-2 \alpha \lambda_{i} \Gamma\right) \Phi_{i} .
\end{aligned}
$$


Selecting $\alpha \geq \frac{1}{\lambda_{1}}$, the expression of $\dot{V}$ reduces to

$\dot{V} \leq \sum_{i=1}^{N} \lambda_{i} \Phi_{i}^{T}\left(P A+A^{T} P-P B R^{-1} B^{T} P+\mu P P+\frac{\eta^{2}}{\mu}\right) \Phi_{i}$.

This implies $\dot{V} \leq 0$ if (6) holds. Therefore, we have $\lim _{t \rightarrow \infty} \Phi(t)=0$ and

$$
\lim _{t \rightarrow \infty} \delta(t)=0
$$

via Barbalat's lemma. Hence, the robust formation of the robot swarm can be achieved. This completes the proof.

Remark 1: The iterations of this algorithm is proportional to the number of robots, such that the computational complexity of this algorithm is $O(N)$. Hence, modern embedded processors can efficiently perform this task and thus the proposed strategy can be easily implemented in the real applications.

\section{Simulations With unmanned AERial Vehicles}

In this section, a target surveillance mission was performed by a team of tricopter UAVs. The configuration of the tricopter UAV was analyzed in [18], where the three rotors of this aerial robotic platform can be tilted independently to get complete force and torque vectoring authority. The entire simulation study has been performed in the Matlab-Simulink environment using Simscape Multibody toolbox, which is used to model the UAV realistically. Refer to [18] for more details about the UAV dynamic model and the simulation settings. Perturbation due to wind was added along the $x$-axis to verify the robustness of the UAV swarm in extreme conditions.

For this case study, all the twelve UAVs were expected to attain a time-varying dodecagon formation surrounding the target for monitoring the full range of target activity. Fig. 2 presents the three-dimensional virtual reality pictures of the spatial position of the UAVs at different time instant during the target monitoring mission. The attitude and position responses of the UAVs are shown in Fig. 3. It can be seen that the roll, pitch and yaw orientation of the UAVs have converged to zero at the steady-state condition. Fig. 4(a) portrays the cumulative spatial position trajectories of all twelve UAVs in the threedimensional plane during the mission staring from their initial positions. To show the superior performance of the proposed coordination strategy, the same mission was also performed using the conventional swarm controller developed in [18] for comparison. The formation tracking errors of both the controllers during the target surveillance mission are illustrated in Fig. 4(b), it can be easily found that the proposed method leads to a more stable and accurate output tracking response under the model uncertainties and external disturbances in the environment.

\section{EXPERIMENTAL VALIDATION WITH MOBILE ROBOTS}

To investigate the performance of the proposed formation scenario, we used a collection of real robots, namely Mona robots which are an open-source swarm robotic platform [19]. The robot is based on Arduino AVR architecture with ATMEGA-328 micro-controller. It is actuated with two wheels (with $3.2 \mathrm{~mm}$ diameter), which are differentially driven

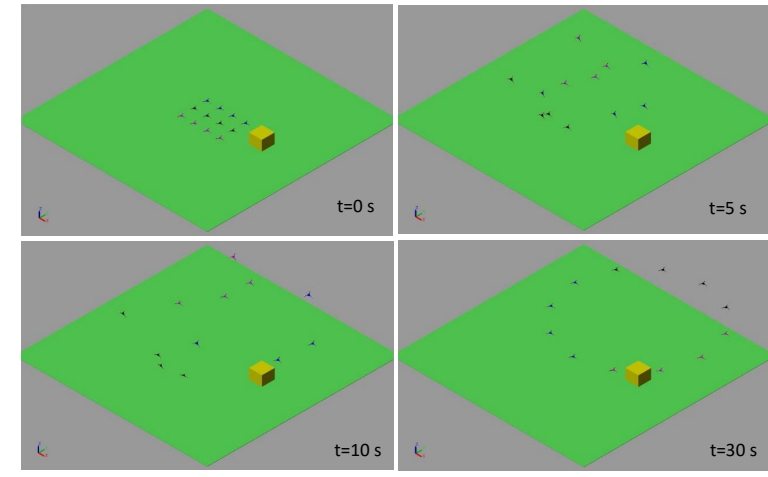

Fig. 2. Position snapshots of the 12 UAVs and the target at different time instants.
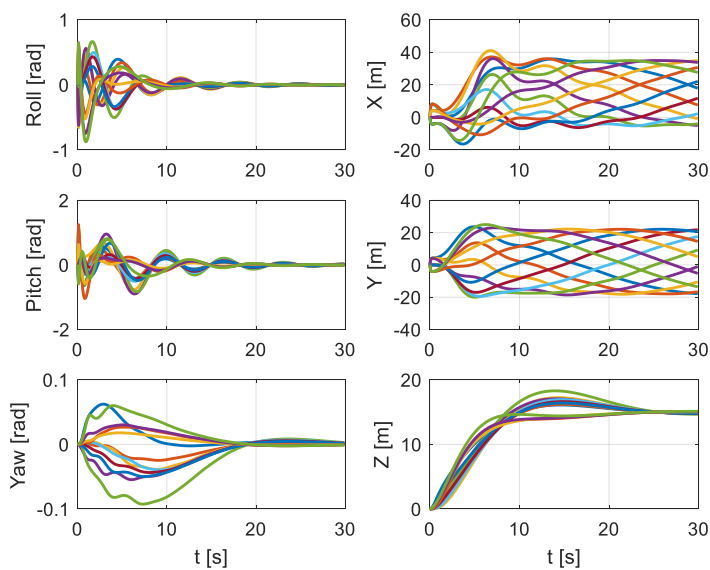

Fig. 3. Attitude and position responses of the swarm system.

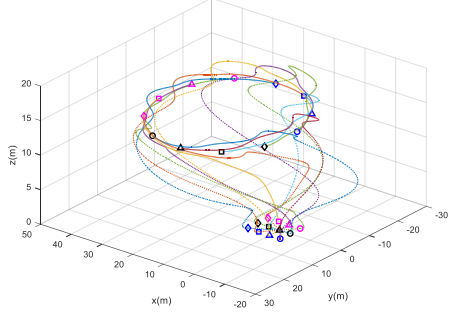

(a)

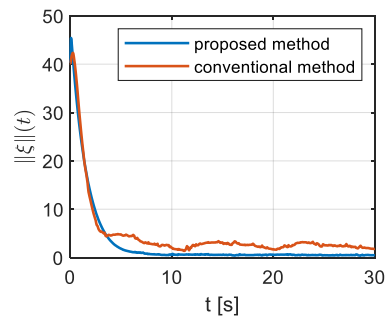

(b)
Fig. 4. (a) Position trajectories of the UAVs in the X-Y-Z plane achieving the time-varying formation during the mission. (b) Error tracking performance comparison of the proposed method with the conventional controller in [18].

using two gear-head micro DC motors. The main controller uses PWM (pulse-width modulation) to adjust the rotational speed of the motors independently. In the experimental setup, we used a low-cost Microsoft LifeCam Studio Webcam as the swarm localization platform. The position of each robot was continuously tracked by an open-source tracking software developed in [20] with a sampling time of $0.1 \mathrm{~s}$. A time delay of $0.05 \mathrm{~s}$ and a tracking error of $\pm 0.005 \mathrm{~m}$ can be observed during the experiments due to the processing speed of the host computer and the quality of the camera. Hence, the robustness of the swarm system subjected to certain communication delays, actuator noises and inaccuracies of the camera tracking system can be verified via the experiments. 


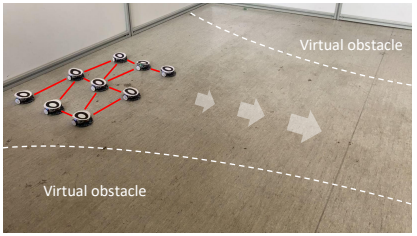

(a)

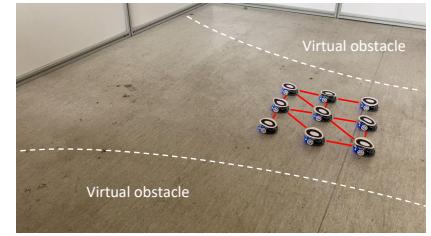

(b)
Fig. 5. Position snapshots of the mobile robots: (a) $t=0 \mathrm{~s}$. (b) $t=60 \mathrm{~s}$.

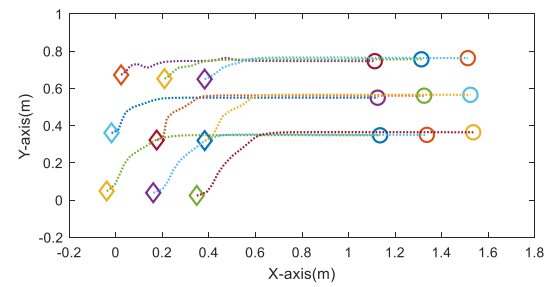

Fig. 6. Position trajectories of the mobile robots achieving the collective motion. The initial positions of the robots are represented by diamonds and the final positions of the robots are represented by circles.

All the mobile robots used in the experiments have the same physical and hardware configuration. The dynamic model in terms of the global coordinates can be described as follows

$$
\dot{p}_{x i}=v_{i} \cos \theta_{i}, \dot{p}_{y i}=v_{i} \sin \theta_{i}, \dot{\theta}_{i}=\omega_{i},
$$

where $\left(p_{x i}, p_{y i}\right)$ is the position of the $i^{\text {th }}$ robot in X-Y plane and $\theta_{i}$ is the orientation. $v_{i}$ and $\omega_{i}$ represent the linear and angular velocities of the $i^{\text {th }}$ robot respectively.

In this real-time experiment, the nine robots were expected to maintain a square formation based on local information while following a moving guidance signal in the virtual cluttered environment. Position snapshots of the initial positions and final positions in the experiment are provided in Fig. 5(a) and Fig. 5(b), respectively, where the communication topology among the robots are represented by the red lines. The trajectories of all the robots during the task are illustrated in Fig. 6. It can be concluded that the proposed control protocol has been able to successfully accomplish the real-time experiments involving wheeled mobile robots under certain disturbances in the lab environment such as actuator noises and communication delays.

The video containing the simulation and experiment results is provided in the Supplementary Material.

\section{CONCLUSIONS}

In this brief, a novel collective formation coordination strategy was proposed for nonlinear robot swarms with unknown disturbances. All the robots connected by a communication network were able to track a dynamic target with uncertain maneuvers. A method to design the control law was given and the convergence of the swarm system was proved through the Lyapunov stability theory. Simulations and real-time hardware experiments were performed to validate the robustness and feasibility of the proposed coordination approach. In the future, fault-tolerant method, such as [21], will be integrated with the proposed coordination design to deal with hardware damage in the extreme environments.

\section{REFERENCES}

[1] M. Dorigo, G. Theraulaz, and V. Trianni, "Reflections on the future of swarm robotics," Science Robotics, vol. 5, p. eabe4385, 2020.

[2] M. Brambilla, E. Ferrante, M. Birattari, and M. Dorigo, "Swarm robotics: a review from the swarm engineering perspective," Swarm Intelligence, vol. 7, no. 1, pp. 1-41, 2013.

[3] J. Hu, A. E. Turgut, T. Krajnik, B. Lennox, and F. Arvin, "Occlusionbased coordination protocol design for autonomous robotic shepherding tasks," IEEE Transactions on Cognitive and Developmental Systems, 2020, DOI:10.1109/TCDS.2020.3018549.

[4] Y. Liu, P. Shi, and C.-C. Lim, "Collision-free formation control for multiagent systems with dynamic mapping," IEEE Transactions on Circuits and Systems II: Express Briefs, vol. 67, no. 10, pp. 1984-1988, 2020.

[5] J. Hu, H. Niu, J. Carrasco, B. Lennox, and F. Arvin, "Voronoi-based multi-robot autonomous exploration in unknown environments via deep reinforcement learning," IEEE Transactions on Vehicular Technology, vol. 69, no. 12, pp. 14 413-14 423, 2020.

[6] S. Li and Y. Guo, "Distributed source seeking by cooperative robots: Allto-all and limited communications," in IEEE International Conference on Robotics and Automation, 2012, pp. 1107-1112.

[7] S. Li, Y. Guo, and B. Bingham, "Multi-robot cooperative control for monitoring and tracking dynamic plumes," in IEEE International Conference on Robotics and Automation, 2014, pp. 67-73.

[8] J. Hu, P. Bhowmick, I. Jang, F. Arvin, and A. Lanzon, "A decentralized cluster formation containment framework for multi-robot systems," IEEE Transactions on Robotics, in press, 2021.

[9] A. H. Khan, S. Li, and X. Luo, "Obstacle avoidance and tracking control of redundant robotic manipulator: An RNN-based metaheuristic approach," IEEE Transactions on Industrial Informatics, vol. 16, no. 7, pp. 4670-4680, 2019.

[10] A. T. Khan, S. Li, and X. Cao, "Control framework for cooperative robots in smart home using bio-inspired neural network," Measurement, vol. 167, p. 108253, 2021.

[11] X. Dong, Y. Zhou, Z. Ren, and Y. Zhong, "Time-varying formation tracking for second-order multi-agent systems subjected to switching topologies with application to quadrotor formation flying," IEEE Transactions on Industrial Electronics, vol. 64, no. 6, pp. 5014-5024, 2016.

[12] J. Hu, P. Bhowmick, F. Arvin, A. Lanzon, and B. Lennox, "Cooperative control of heterogeneous connected vehicle platoons: An adaptive leaderfollowing approach," IEEE Robotics and Automation Letters, vol. 5, no. 2, pp. 977-984, 2020.

[13] P. Rao and X. Li, "Cooperative formation of self-propelled vehicles with directed communications," IEEE Transactions on Circuits and Systems II: Express Briefs, vol. 67, no. 2, pp. 315-319, 2020.

[14] K. Wu, J. Hu, B. Lennox, and F. Arvin, "Finite-time bearing-only formation tracking of heterogeneous mobile robots with collision avoidance,' IEEE Transactions on Circuits and Systems II: Express Briefs, 2021, DOI:10.1109/TCSII.2021.3066555.

[15] Y. Gong, G. Wen, Z. Peng, T. Huang and Y. Chen, " ObserverBased Time-Varying Formation Control of Fractional-Order Multi-Agent Systems With General Linear Dynamics,' IEEE Transactions on Circuits and Systems II: Express Briefs, vol. 67, no. 1, pp. 82-86, 2020.

[16] J. Hu, P. Bhowmick, and A. Lanzon, "Two-layer distributed formationcontainment control strategy for linear swarm systems: Algorithm and experiments," International Journal of Robust and Nonlinear Control, vol. 30, no. 16, pp. 6433-6453, 2020.

[17] J. Sun and Z. Geng, "Adaptive consensus tracking for linear multiagent systems with heterogeneous unknown nonlinear dynamics," International Journal of Robust and Nonlinear Control, vol. 26, no. 1, pp. 154-173, 2016.

[18] J. Hu and A. Lanzon, "An innovative tri-rotor drone and associated distributed aerial drone swarm control," Robotics and Autonomous Systems, vol. 103, pp. 162-174, 2018.

[19] F. Arvin, J. Espinosa, B. Bird, A. West, S. Watson, and B. Lennox, "Mona: an affordable open-source mobile robot for education and research," Journal of Intelligent \& Robotic Systems, vol. 94, no. 3-4, pp. 761-775, 2019.

[20] T. Krajnik, M. Nitsche, J. Faigl, P. Vanek, M. Saska, L. P $`$ reucil, T. Duckett, and M. Mejail, "A practical multirobot localization system," Journal of Intelligent \& Robotic Systems, vol. 76, no. 3-4, pp. 539-562, 2014.

[21] Z. Li, C. Li, S. Li, and X. Cao, "A fault-tolerant method for motion planning of industrial redundant manipulator," IEEE Transactions on Industrial Informatics, vol. 16, no. 12, pp. 7469-7478, 2019. 